\title{
Intraoperative spontaneous renal allograft rupture
}

\author{
Dr Adrienne Wilson, Dr John Preston, Dr Andrej Grajn. \\ Princess Alexandra Hospital, Brisbane, Australia.
}

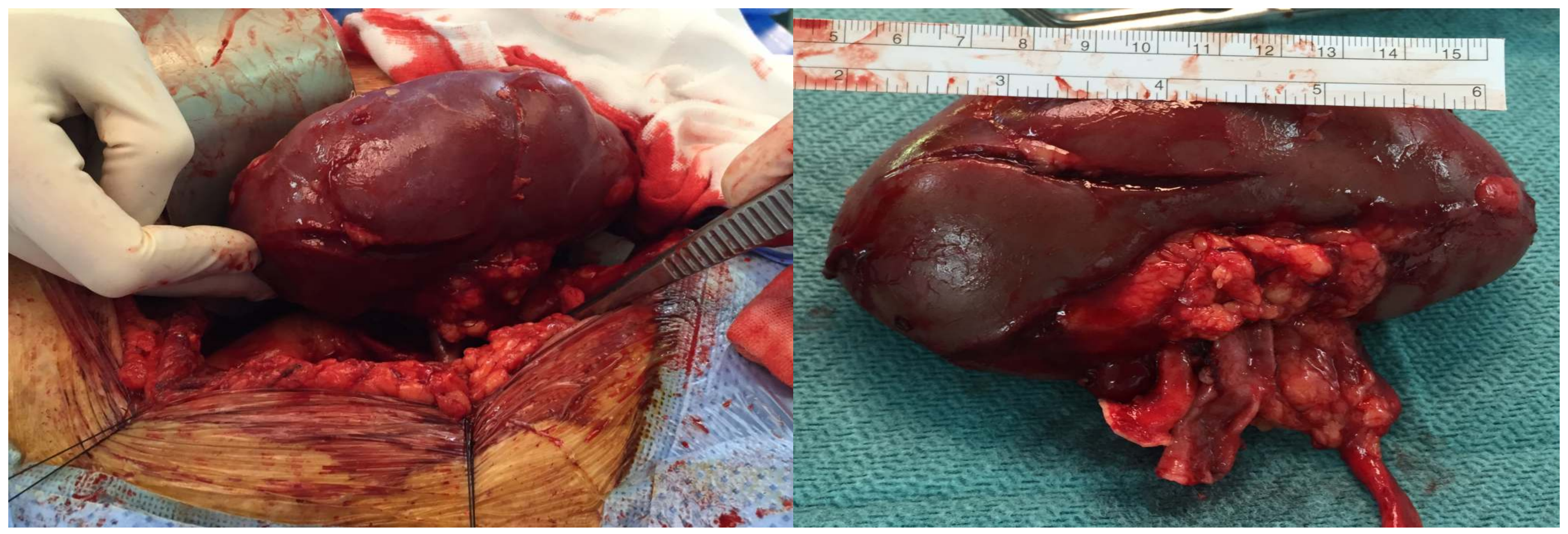

\section{Introduction:}

Spontaneous renal allograft rupture is a rare but serious complication of transplantation. Rupture usually occurs within the first few weeks of surgery and can be caused by acute rejection, acute tubular necrosis (ATN) or renal vein thrombosis. This case report demonstrates spontaneous renal allograft rupture immediately after reperfusion.

\section{Case:}

A 53 year old male received a DBD renal transplant. He had CKD secondary to dysplastic kidney / reflux nephropathy and a previous right nephrectomy for complicated pyelonephritis.

The donor was a 27 year old male who died from hypoxic brain injury after a drug overdose. He became anuric and developed rhabdomyolysis.

Pre-transplant biopsy of the sister kidney showed moderate ATN, but no cortical necrosis. The donor kidney was placed on the LifePort machine overnight for 12 hours. The resistance was high $(0.52 \mathrm{mmHg} / \mathrm{ml} / \mathrm{min})$ and the flow rate was lower than normal $(47 \mathrm{ml} / \mathrm{min})$.

After reperfusion the allograft became tense and pulsatile. The patient became hypertensive and tachycardic. A $65 \mathrm{~mm}$ longitudinal laceration developed and a transplant nephrectomy was performed. He was discharged home on day four with a two week course of immunosuppression and has been re-listed for transplant.

Histopathology of the transplant nephrectomy showed severe ATN and widespread rhabdomyolysis. There was no evidence of renal vein thrombosis.

\section{Discussion:}

Intraoperative spontaneous rupture of the renal allograft may be due to increased intrarenal pressure caused by microvascular injury from ATN and rhabdomyolysis.
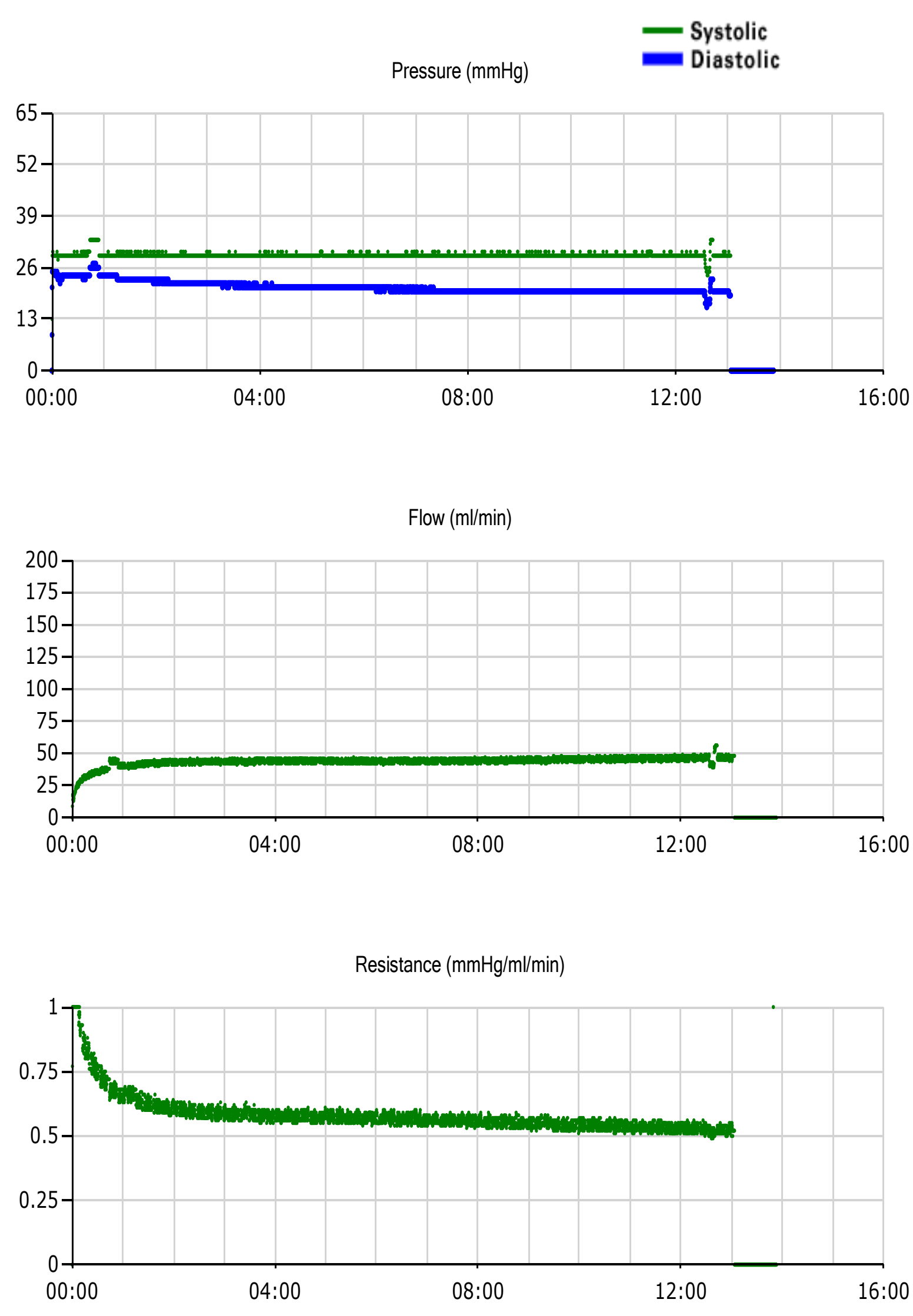

Figure 1: Lifeport perfusion data demonstrating high resistance and low flow in the donor kidney. 